\title{
Quantifying the impact of pump noise on fiber-optic nonlinear processes
}

\author{
Lægsgaard, Jesper
}

Published in:

Journal of the Optical Society of America B: Optical Physics

Link to article, DOI:

10.1364/JOSAB.418236

Publication date:

2021

Document Version

Publisher's PDF, also known as Version of record

Link back to DTU Orbit

Citation (APA):

Lægsgaard, J. (2021). Quantifying the impact of pump noise on fiber-optic nonlinear processes. Journal of the Optical Society of America B: Optical Physics, 38(5), 1538-1545. https://doi.org/10.1364/JOSAB.418236

\section{General rights}

Copyright and moral rights for the publications made accessible in the public portal are retained by the authors and/or other copyright owners and it is a condition of accessing publications that users recognise and abide by the legal requirements associated with these rights.

- Users may download and print one copy of any publication from the public portal for the purpose of private study or research.

- You may not further distribute the material or use it for any profit-making activity or commercial gain

- You may freely distribute the URL identifying the publication in the public portal

If you believe that this document breaches copyright please contact us providing details, and we will remove access to the work immediately and investigate your claim. 


\title{
Quantifying the impact of pump noise on fiber-optic nonlinear processes
}

\author{
JESPER L/EGSGAARD (1) \\ DTU Fotonik, Department of Photonics Engineering, Technical University of Denmark, Ørsteds Plads 343, DK-2800 Kongens Lyngby, Denmark \\ (jlag@fotonik.dtu.dk)
}

Received 21 December 2020; revised 27 February 2021; accepted 21 March 2021; posted 23 March 2021 (Doc. ID 418236); published 14 April 2021

\begin{abstract}
The impact of pump pulse fluctuations on ultrafast nonlinear frequency conversion processes in optical fibers is investigated. A simple measure of the sensitivity of a particular process to pump technical noise is introduced and tested against numerical ensemble simulations for normal-dispersion continuum generation and dispersive-wave generation. The proposed measure is found to adequately describe the impact of technical noise at the per mill or percent level. (0) 2021 Optical Society of America
\end{abstract}

https://doi.org/10.1364/JOSAB.418236

\section{INTRODUCTION}

Nonlinear frequency conversion in optical fibers has become an important tool for expanding the wavelength range of fiberbased laser systems [1-4] and facilitating applications requiring broadband sources such as optical coherence tomography [5]. Current commercial broadband, or "supercontinuum" sources, typically inject picosecond pump pulses into carefully engineered microstructured optical fibers (MOFs) to generate broadband spectra through a complex cascade of nonlinear processes $[1,6]$. As is well known, these sources suffer from high relative intensity noise due to the initial breakup of the pump pulse being seeded by high-frequency quantum noise [7]. This fundamental noise source is exacerbated by the complex and turbulent nature of the ensuing nonlinear processes $[8,9]$. An established route to low-noise sources is to launch pump pulses with lower soliton number (essentially shorter and less energetic pulses) $[6,10]$ and to utilize simpler nonlinear processes, such as self-phase modulation (SPM) [4] or dispersive-wave generation (DWG) $[2,11]$. Until now, such light sources have mostly been demonstrated in research laboratories; however, they can be expected to become commercially important with the continuing development of femtosecond $(\mathrm{fs})$ fiber lasers, which in practice are needed to obtain useful conversion efficiencies.

While the amplification of unavoidable quantum noise fluctuations can be avoided by these means, recent investigations of supposedly "noise-free" sources have revealed that practical implementations in fact suffer from significant output noise, due to the sensitivity of the nonlinear processes to pump pulse fluctuations $[12,13]$. Although some degree of technical noise is always to be expected, in numerical investigations of nonlinear frequency conversion it is often overlooked. This is because inclusion of this noise source in simulations would depend on assumptions specific to a particular pump source, and so the results might not be generally valid. With the ongoing development of low-noise sources, there is a need to quantify the impact of technical noise on specific nonlinear processes in a general way. While technical noise may in general be understood as any noise contribution that is not fundamental in nature (i.e., not caused by quantum fluctuations), in this contribution the focus will be on noise that can be described in terms of one or a few fluctuating parameters.

The purpose of this paper is to suggest a straightforward, even simple-minded, measure of pump noise sensitivity and investigate its applicability in a couple of important examples, namely, dispersive wave and/or continuum generation in fibers with either anomalous or normal dispersion. It will be shown that the relative intensity noise appearing in the spectral output of ensemble simulations incorporating practically relevant levels of pump technical noise can be predicted based on simple sensitivity functions, which can be evaluated from only a few simulations. Furthermore, these functions can be used to estimate the level of pump noise that is meaningful to target in light of the presence of unavoidable quantum noise. This methodology could aid in the further development of low-noise light sources based on nonlinear processes into practically useful devices.

\section{FORMAL THEORY}

Consider an observable quantity, $S$, which is the output of some nonlinear optical process whose input is a pump pulse specified by a set of parameters $p$. In the present paper, $S$ will be the frequency-dependent spectral energy density; in principle, however, it could also be other quantities, e.g., the integral of spectral energy density over some frequency interval, the peak power of a compressed output pulse, etc. Similarly, the set of 
parameters $p$ will in this investigation be taken to be peak power and duration of the pump pulse, but other defining parameters could also be considered. An important requirement is that the parameters should be independently variable, e.g., if power and duration are considered, pulse energy could not be added as a third parameter. Similarly, a transform-limited pulse duration and bandwidth could not be considered independent, whereas it would be possible for a chirped pulse. The choice of pulse model and parameters must be informed by both the expected properties of pump sources and the properties of the nonlinear process. As an example, whereas most practical pump sources would be expected to exhibit some amount of timing jitter, this has no influence on the output spectrum of a single-pass nonlinear process as long as consecutive pump pulses are well separated in time throughout the propagation. On the other hand, for a process involving back-seeding, such as a parametric oscillator, timing jitter might contribute to spectral fluctuations.

In a sequence of $M$ simulations/experiments with different realizations, the parameter values in a given instance $m$ are given by

$$
p_{m}=p_{0}+\delta p_{m},
$$

where $p_{0}$ is the average value, and $\delta p_{m}$ is a distribution with zero mean, and relative fluctuation $R_{p}$ is defined by

$$
R_{p}=\frac{\sqrt{\left\langle\delta p_{m}^{2}\right\rangle}}{p_{0}} \equiv \frac{\delta p}{p_{0}} .
$$

The brackets denote an average over the ensemble, i.e.,

$$
\left\langle S_{m}\right\rangle=\frac{1}{M} \sum_{m} S_{m},
$$

etc.

The pump fluctuations will lead to a relative fluctuation of $S$ (relative intensity noise in the case where $S$ is a spectral energy density function as a function of wavelength or frequency) given by

$$
R_{S} \equiv \frac{\delta S}{S_{0}}=\frac{\sqrt{\left\langle S_{m}^{2}\right\rangle-\left\langle S_{m}\right\rangle^{2}}}{\left\langle S_{m}\right\rangle} \approx \frac{\sqrt{\sum_{p}\left(\frac{\partial S}{\partial p}\right)^{2} \delta p^{2}+\delta S_{q}^{2}}}{S_{0}},
$$

where the last approximate equality is accurate through the second order in the $\delta p$. Here, $\delta S_{q}$ is the "quantum" fluctuation of $S$, i.e.,

$$
\delta S_{q}=\sqrt{\left\langle S_{m}^{2}\right\rangle-\left\langle S_{m}\right\rangle^{2}},
$$

where the ensemble average is over realizations without pump fluctuations but with fundamental (quantum) noise seeding [14]. This result is well-known from the elementary theory of measurement uncertainties. For completeness, a brief derivation is given in Appendix A. Introducing the dimensionless quantities

$$
S_{p} \equiv \frac{p_{0}}{S_{0}} \frac{\partial S}{\partial p}
$$

Eq. (4) may be rewritten as

$$
R_{S}=\sqrt{\sum_{p} S_{p}^{2} R_{p}^{2}+R_{S q}^{2}} ; \quad R_{S q}=\frac{\delta S_{q}}{S_{0}} .
$$

The set of dimensionless $S_{p}$ factors, which in the following will be termed parameter, or $p$, sensitivities, is the measure proposed for quantification of pump noise sensitivity. They express how a relative fluctuation in pulse parameters impress a relative fluctuation on the output. For instance, if $\left|S_{p}\right|>1$, input noise in the parameter $p$ is amplified in the output signal, whereas $\left|S_{p}\right|<1$ corresponds to a suppression of noise fluctuations.

If $S_{p} R_{p} \gg R_{S q}$ for one or more pump parameters $p$, then fluctuations in this/these parameters will form the dominant contribution to output noise. Conversely, if all pump parameters satisfy $S_{p} R_{p} \ll R_{S q}$, quantum noise will dominate. Therefore, the relative pump noise level

$$
R_{p q} \equiv\left|\frac{R_{S q}}{S_{p}}\right|
$$

can be taken as a measure of the relative noise level one could reasonably strive to obtain in a given pump parameter. In the following, $R_{p q}$ will be denoted the parameter, or $p$, quantum equivalence fluctuation. Reduction of $R_{p}$ below $R_{q p}$ would only lead to a marginal gain in output noise as one would move into a regime dominated by quantum noise.

For some pump sources, pulse parameters, which can in principle be considered independent such as power and duration, are in fact correlated due to the inner workings of the pump laser [12]. For instance, solitonic pulses would have a connection between power $P$ and duration $t$ of the form

$$
P_{m}=\frac{C}{t_{m}^{2}}
$$

Other types of correlations may be present in practical fs lasers [12]. For two parameters, $p$ and $r$, one can in general write

$$
r_{m}=f\left(p_{m}\right) \Rightarrow \delta r_{m}=D_{r p} \delta p_{m} ; \quad D_{r p}=\frac{d f}{d p}
$$

for small $\delta p_{m}, \delta r_{m}$. A solitonic pulse would then obey $\delta P_{m}=-2 \frac{C}{t_{0}^{3}} \delta t_{m}$ or $\frac{\delta P_{m}}{P_{0}}=-2 \frac{\delta t_{m}}{t_{0}}$. The expression for the relative signal fluctuation $R_{S}$ should now be modified to

$$
R_{S}=\sqrt{\left(S_{p}+D_{r p} \frac{p_{0}}{r_{0}} S_{r}\right)^{2} R_{p}^{2}+R_{S q}^{2}},
$$

which, in the example of the solitonic pulse, would lead to the expression

$$
R_{S}=\sqrt{\left(S_{t}-2 S_{P}\right)^{2} R_{t}^{2}+R_{S q}^{2}} .
$$

Thus, if the pump duration and power sensitivities $S_{t}, S_{P}$ have the same sign, some degree of cancellation between fluctuations caused by power and duration might be expected, but not if they have an opposite sign. 


\section{NUMERICAL RESULTS}

\section{A. Simulation Methods}

In this section, the merits of the proposed pump noise measure $S_{p}$ will be investigated through numerical simulations of nonlinear frequency conversion processes, which are supposedly "deterministic" or "low-noise" in the sense that quantum noise seeds have a minimal impact on the output. Specifically, supercontinuum generation through self-phase modulation and wave breaking in fibers with weak normal dispersion will be considered as well as dispersive-wave generation in fibers with anomalous dispersion.

The nonlinear simulations will consider a single polarization state of a single guided mode in an MOF designed to obtain appropriate dispersion properties. The simulations are done using the formal framework laid out in [15]. The relevant fiber properties, i.e., dispersion and effective areas, are calculated by a full-vectorial finite-difference method, similar to that presented by Zhu and Brown [16]. Input pulses are taken to be transformlimited Gaussian functions, with average peak power $P_{0}$ and average duration (full width at half maximum of the temporal power profile) $t_{0}$. The pump wavelength is always $1.064 \mu \mathrm{m}$, which is a commonly used wavelength in, e.g., Nd:YAG or $\mathrm{Yb}$-doped fiber lasers.

In the noise simulations, the fluctuating parameters are considered to be $P_{0}$ and $t_{0}$. A fluctuation in pump wavelength could straightforwardly be added along the same lines but is not taken into account here. The pump power and duration sensitivities $S_{P}$ and $S_{t}$ are evaluated directly by finite differences as

$$
S_{P}(\lambda)=\frac{S\left(\lambda ; P_{0}(1+\Delta), t_{0}\right)-S\left(\lambda ; P_{0}(1-\Delta), t_{0}\right)}{2 S\left(\lambda ; P_{0}, t_{0}\right) \Delta}
$$

and similarly for $S_{t}$. Here, $S(\lambda ; P, t)$ is the output spectrum as a function of $P$ and $t$, and $\Delta$ is a dimensionless finite-differencing parameter, which is chosen as $10^{-3}$ in this work. Thus, a total of five simulations are needed to set up the parameter sensitivities $S_{p}$ for two parameters; in general, $2 N+1$ simulations will be needed for a set of $N$ parameters. These simulations are performed without quantum noise seeding. In addition, to evaluate the relative quantum fluctuations $R_{S q}$, an ensemble simulation with fixed pump parameters but one-photon quantum noise added to the input spectrum is performed. From these results, relative intensity noise in the presence of various levels of pump noise can be predicted according to Eq. (7). These predictions are compared with results from ensemble simulations with both quantum noise seeding and normally distributed noise in the pump $P$ and $t$ parameters.

\section{B. Normal-Dispersion Continuum Generation}

Supercontinuum generation by self-phase modulation can be effective if the dispersion coefficient $D$ is small, to prevent rapid pulse broadening, and negative (normal), to prevent pulse collapse and/or modulational instability. MOFs provide a technological opportunity to tailor the desired dispersion curves [17]. The inset in Fig. 1 shows a typical design, with a triangular array of airholes whose diameter is 0.39 times their separation distance, or pitch, $\Lambda$. The main panel of Fig. 1 shows

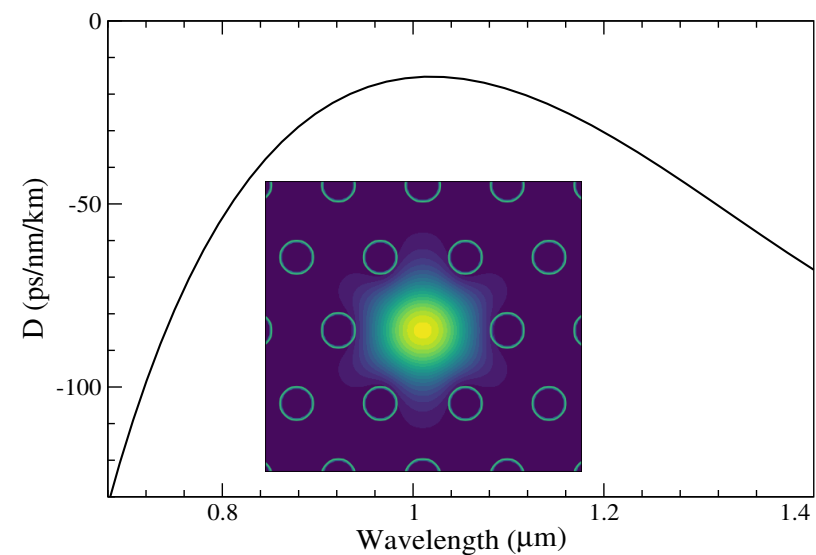

Fig. 1. Fundamental mode intensity at the pump wavelength in the normal-dispersion fiber (inset) and dispersion coefficient $D$ versus wavelength (main).

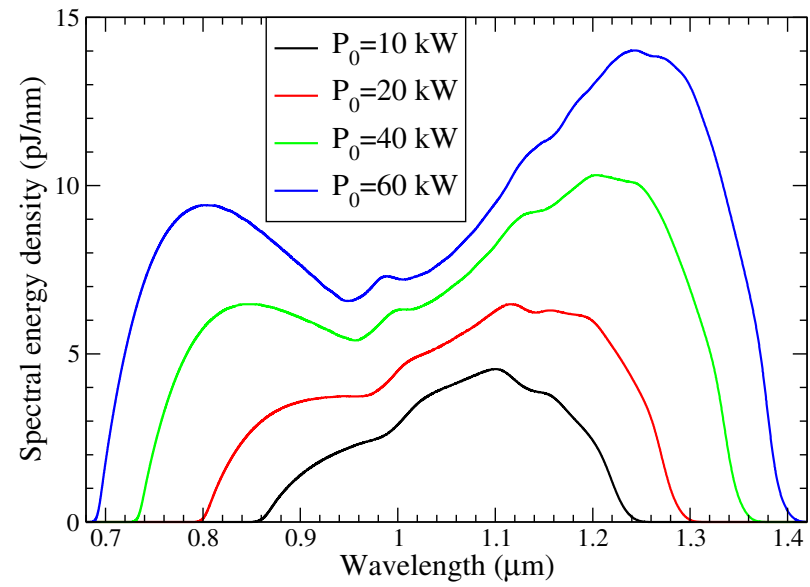

Fig. 2. Spectral evolution with power for normal-dispersion continuum generation in $60 \mathrm{~cm}$ of fiber and a fixed pulse duration of $100 \mathrm{fs}$.

the dispersion curve for a pitch of $1.45 \mu \mathrm{m}$, which is the design considered in the numerical simulations.

In Fig. 2, the spectral evolution versus pump power is shown. The pulse duration is fixed at $100 \mathrm{fs}$, and the length of the fiber is $60 \mathrm{~cm}$, since there is little spectral change occurring beyond this length. Increasing power leads to a broader spectrum, with higher spectral energy density, steeper edges, and more structure in the central part of the spectrum.

In Fig. 3, the pump duration and power sensitivities $S_{t}, S_{P}$ as well as $S_{t}-2 S_{P}$ are plotted for $P_{0}=20 \mathrm{~kW}$ and $60 \mathrm{~kW}$. Apart from the broadening of the spectrum, the two cases appear very similar. The pump duration sensitivity $S_{t}$ has a value around 1 in the central part of the spectrum, whereas the power sensitivity $S_{P}$ reaches somewhat lower values of around 0.3 . This may be understood by noting that an increase in $t$ will increase the pulse energy while reducing the SPM-induced broadening. Both of these effects will increase the energy density in the central part of the spectrum. Increasing $P$, on the other hand, will increase pulse energy but also spectral broadening, which pulls in opposite directions with regard to the central spectral energy density. In the wings of the spectrum, on the other hand, an 

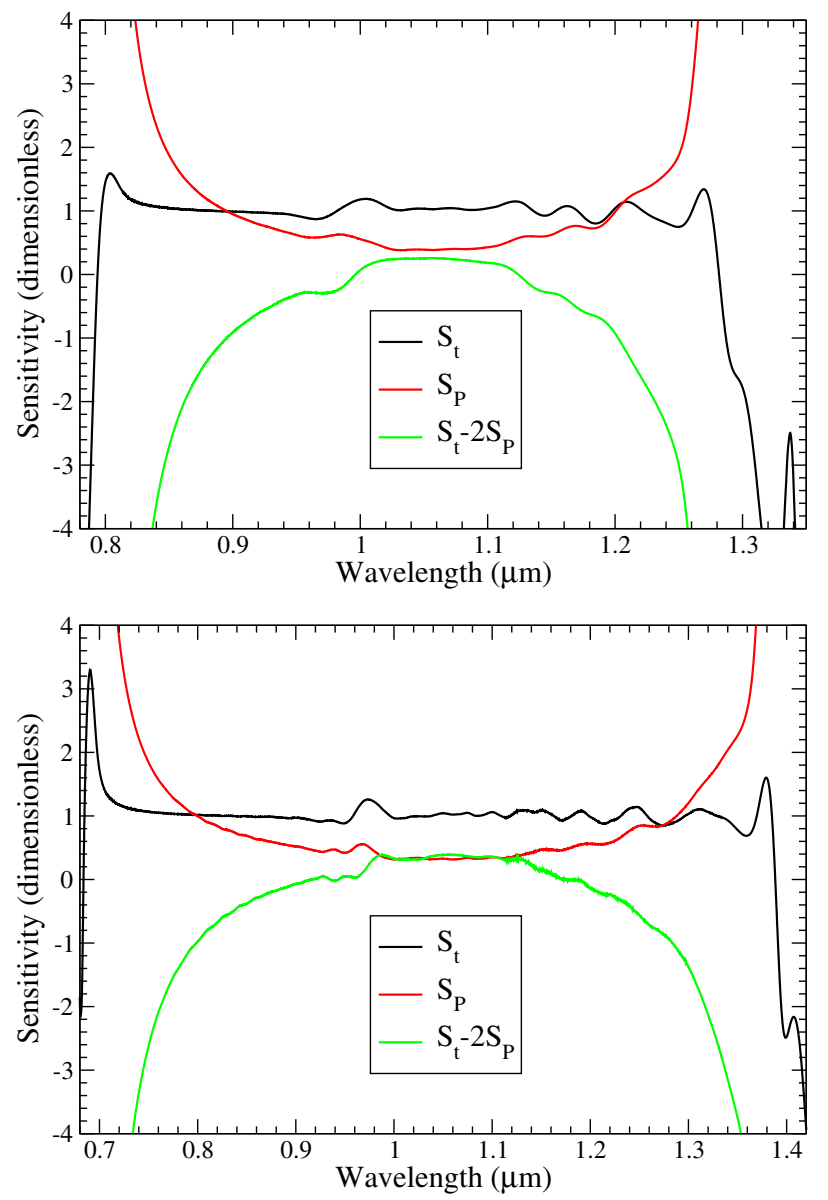

Fig. 3. Pump duration and power sensitivities $S_{t}$ and $S_{P}$ for $P_{0}=20 \mathrm{~kW}$ (top) and $60 \mathrm{~kW}$ (bottom), and $t_{0}=100 \mathrm{fs}$. Also shown is the combination $S_{t}-2 S_{P}$, which describes the total sensitivity to pump pulse duration when this parameter correlates to pulse power as in Eq. (9).

increase in $P$ leads to an increase in energy density both due to stronger broadening and higher pulse energy, and so $S_{P}$ rises strongly toward the edges of the spectrum, whereas $S_{t}$ stays almost constant until the very edge. Thus, in the central part of the spectrum, fluctuations in pulse duration can be expected to dominate the (technical) noise, whereas toward the edges, peak power fluctuations become the dominant noise source. It can also be noted that the combined function $S_{t}-2 S_{p}$, which, as discussed above, is relevant for pump sources, where $P$ and $t$ are correlated according to a solitonic balance condition, becomes quite low in the central part, around 0.2-0.3; in this range, such a source will be advantageous with respect to noise. This does not hold, however, toward the edges, where the factor of 2 in front of $S_{P}$ leads to a stronger increase than in the case of uncorrelated $P, t$ parameters.

The validity of Eq. (7) rests on an expansion of the spectral energy density to second order in the pump parameter fluctuations. It is, then, of interest if this expansion will be reliable for reasonable levels of pump fluctuations. In Fig. 4, the relative signal fluctuation $R_{S}$ for a pump power of $60 \mathrm{~kW}$ is plotted together with the normalized spectrum for relative fluctuations in $P, t$ of $0.1 \%$ (top panel) and 5\% (bottom panel) and compared with the predictions of Eq. (7) with and without the
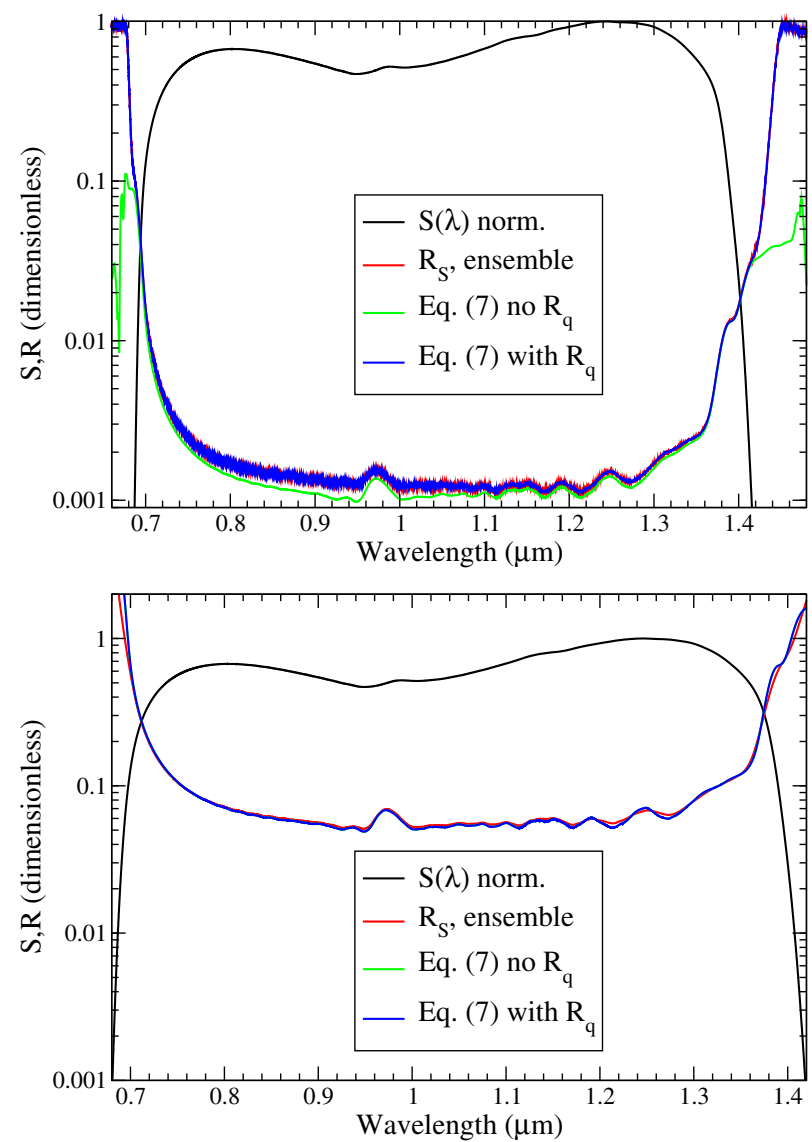

Fig. 4. Normalized spectral energy density (black), numerically calculated relative signal fluctuation $R_{S}(\mathrm{red})$, and $R_{S}$ predictions from Eq. (7) with and without the relative quantum fluctuation $\left(R_{S q}\right)$ term describing quantum noise included. Results are shown for $P_{0}=60 \mathrm{~kW}$ and independent relative pump power and duration fluctuations $R_{P}=R_{t}=10^{-3}$ (top) and $5 \cdot 10^{-2}$ (bottom).

relative quantum fluctuation term $R_{S q}$ included. The numerical $R_{S}$ curves were obtained from ensembles of 1000 simulations. For the small fluctuation, Eq. (7) excellently predicts $R_{S}$, but it can be noted that neglect of $R_{S q}$ leads to a noticeable deviation, implying that quantum noise begins to play a role at this level of pump fluctuations. For the 5\% pump fluctuation, quantum noise is of no consequence. Further, in this case the predictions of Eq. (7) are useful, although deviations of a few percent are seen. As expected from the pump duration and power sensitivity curves $S_{t}$ and $S_{P}$ in Fig. 3, the relative fluctuations in the central part of the spectrum are slightly larger than those of the pump but become stronger toward the edges of the spectrum. These findings are in good agreement with the results of [12].

In Fig. 5, a corresponding comparison is shown for a pump whose parameters are correlated according to Eq. (9), i.e., pulses shaped by soliton dynamics. The relative fluctuation of pump pulse duration was $5 \%$. As expected, the spectral fluctuations in the central wavelength range are lower, around $2 \%$, and become very low in two distinct spectral ranges, due to zero crossings of the combined sensitivity $S_{t}-2 S_{P}$ for solitonic pulses, as seen in Fig. 3. In these regions, there are some deviations between the ensemble simulation and the predictions of Eq. (7), showing 


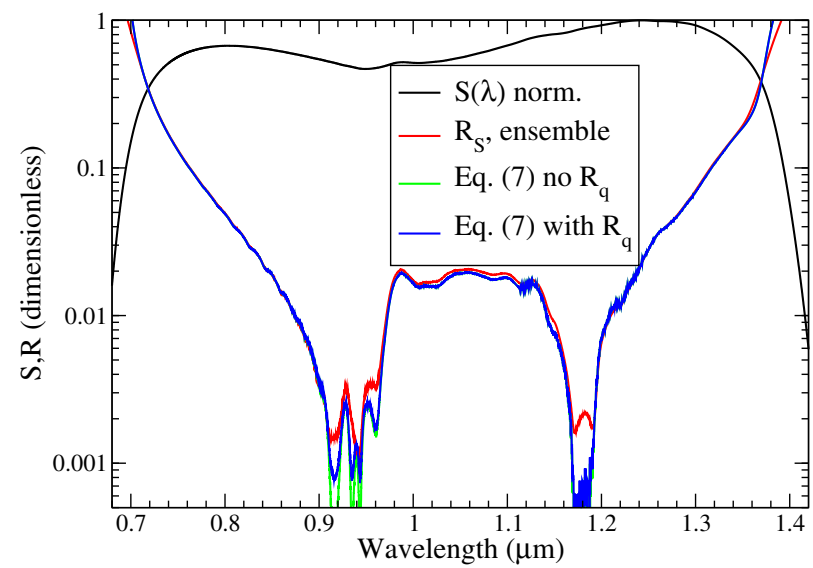

Fig. 5. Same as Fig. 4, except that the $P, t$ pump pulse parameters are correlated according to a soliton balance condition. The relative pump duration fluctuation $R_{t}=5 \cdot 10^{-2}$.

that higher-order terms in the pump fluctuation become significant. However, the qualitative prediction of strongly reduced noise in these regions is borne out by the simulation. As before, the strong increase in noise at the spectral edges is correctly predicted by Eq. (7).

Figure 6 shows the pump duration and power quantum equivalence fluctuations $R_{t q}$ and $R_{P q}$ defined by Eq. (8) for pump powers of 20 and $60 \mathrm{~kW}$. In both cases, it can be seen that relative pump power and duration fluctuations of $R_{P} \sim R_{t} \sim 10^{-3}$ are desirable. This is in line with the curves in Fig. 4, which showed that quantum noise became noticeable at this level of pump noise.

\section{Dispersive-Wave Generation}

In this subsection, the pump noise's impact on dispersive-wave generation (DWG) in fibers with anomalous dispersion at the pump wavelength is considered. This wavelength-conversion technique has been widely studied as a low-noise alternative to ps-pumped supercontinuum sources pumped in anomalous dispersion, which are inherently noisy. Its sensitivity to pump noise is therefore a question of high importance.

A microstructured fiber design similar to the one shown in Fig. 3 is considered but with an airhole diameter of $0.83 \Lambda$. DWG relies on the self-compression and collapse of an ultrafast pulse due to the interplay of self-phase modulation and anomalous dispersion. During the collapse, the pulse peak may compress to a few fs, resulting in a wide spectral expansion, which only persists around the point of maximal compression. However, power around a certain wavelength, $\lambda_{d}$, may be decoupled from the high-power pulse and released as freely propagating dispersive waves subject to the phase-matching criterion:

$$
\beta\left(\omega_{d}\right)=\beta\left(\omega_{p}\right)+\left(\omega_{d}-\omega_{p}\right) \beta_{1}\left(\omega_{p}\right)+\Gamma_{\mathrm{NL}} .
$$

Here, $\beta$ is the propagation constant of the guided mode, $\omega_{p}$ is the pump angular frequency, $\omega_{d}$ is the angular frequency corresponding to $\lambda_{d}$, and $\beta_{1}=d \beta / d \omega$. $\Gamma_{\mathrm{NL}}$ is a nonlinear phase contribution, which can be estimated as $\frac{\gamma P_{0}}{2}$ for a fundamental soliton with peak power $P_{0}$, continuously emitting dispersive
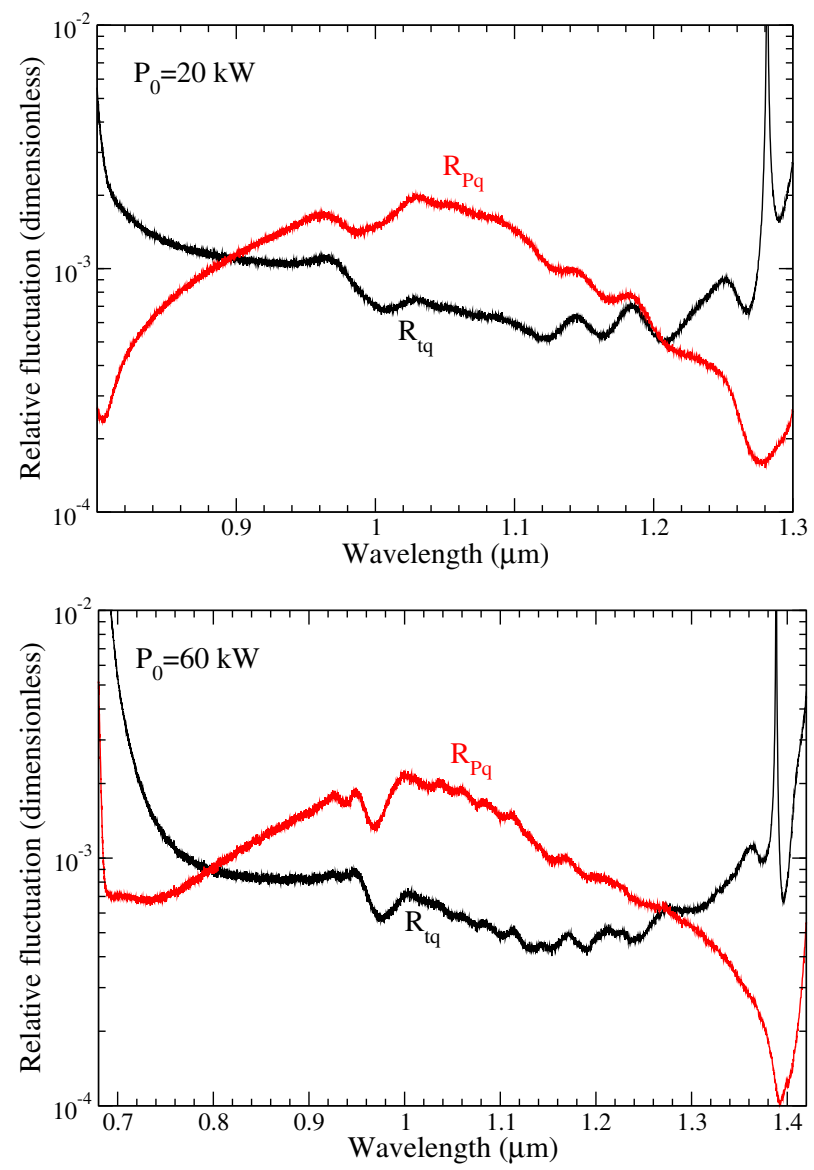

Fig. 6. Pump duration and power quantum equivalence fluctuations $R_{t q}$ and $R_{P q}$ as defined by Eq. (8) for $P_{0}=20 \mathrm{~kW}$ (top) and $60 \mathrm{~kW}$ (bottom).

waves [18], where $\gamma$ is the nonlinear coefficient. For the more common self-compression scheme described here, the value of this nonlinear correction is not so straightforward to estimate [19]. This may in part be due to the fact that the center wavelength of the dispersive pulse undergoes a significant blueshift immediately after its generation, due to cross-phase modulation with the pump pulse until the two pulses separate temporally (the dispersive wave is invariably emitted through the trailing edge of the pump pulse, due to the nature of the phase-matching criterion).

The value of $\Lambda$ determines the fiber dispersion and thereby the dispersive-wave position predicted by the linear part of Eq. (14). The nonlinear frequency shift, noise properties, and conversion efficiency are determined by the pulse properties. As before, a Gaussian input pulse with temporal FWHM of $t_{0}=100 \mathrm{fs}$ is considered. The pulse power is quantified through its "soliton number" $N$ defined by

$$
N^{2}=\frac{\gamma P_{0} \tau^{2}}{\left|\beta_{2}\right|} ; \quad \tau=\frac{t_{0}}{\sqrt{2 \ln 2}},
$$

where $\beta_{2}=d^{2} \beta / d \omega^{2}$ at the pump wavelength [20]. As is well known, there is a gradual transition with increasing $N$ from a deterministic regime for $N$-values around 10 or lower, to an inherently noisy regime at $N=20-30[6,10]$. At the same time, increasing $N$ improves conversion efficiency but also leads 


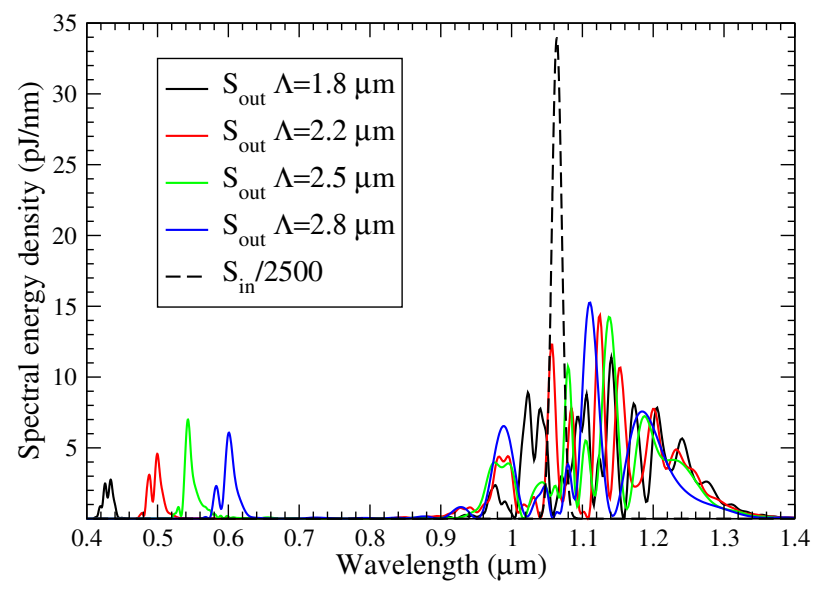

Fig. 7. Spectral energy density $S_{\text {out }}$ after $2 \mathrm{~cm}$ propagation for $N=10$ pulses in fibers with different pitch values. Dashed black line shows the spectrum of the transform-limited Gaussian input pulse, scaled to facilitate comparison.

to a broadening of the DWG peak and eventually continuum formation.

In Fig. 7, spectra from DWG simulations with $N=10$ input pulses in fibers with different $\Lambda$-values are shown. The propagation length is $2 \mathrm{~cm}$. The DWG peak positions predicted from linear phase matching are $0.459 \mu \mathrm{m}, 0.538 \mu \mathrm{m}, 0.599 \mu \mathrm{m}$, and $0.662 \mu \mathrm{m}$ for the four cases in order of increasing $\Lambda$. It can be seen that nonlinear corrections downshift these wavelengths by $25-60 \mathrm{~nm}$. In the following, pump noise impact will be analyzed for $\Lambda=1.8 \mu \mathrm{m}$ and $\Lambda=2.5 \mu \mathrm{m}$, and the main conclusions also pertain to the other cases. In Fig. 8, the pump duration and power sensitivities $S_{t}$ and $S_{P}$ are shown for $N=10$ and $\Lambda=1.8 \mu \mathrm{m}$ and $2.5 \mu \mathrm{m}$. Both derivatives are large and positive on the short-wavelength side of the DWG peak while attaining somewhat smaller and negative values on the long-wavelength side. The DWG spectrum shifts toward shorter wavelengths when increasing either peak power or duration of the input pulse, and both these variations increase the total pulse energy. The combined effect of increased energy and blueshift of the spectrum explains the strong positive derivatives at short wavelengths. On the long-wavelength edge, on the other hand, these effects partly cancel each other, resulting in smaller derivatives with a fluctuating sign.

In both plots, the pump duration sensitivity $S_{t}$ has been multiplied by 2 , and it appears that $2 S_{t} \approx S_{P}$ holds across the spectral range of the DWG pulse. While this is an interesting finding, which seems to hold across a broad range of the calculations performed here, the trend is not reflected in other parts of the spectrum. Closer analysis indicates that the behavior is associated with the nonlinear wavelength shift of the DWG peak, which changes twice as fast for a given relative change of input peak power as for the same relative change of input duration.

In Fig. 9, the relative signal fluctuation $R_{S}$ calculated numerically in a 1000 -simulation ensemble for $\Lambda=2.5 \mu \mathrm{m}, N=10$, and $R_{t}=R_{P}=10^{-3}$ is compared with that predicted by Eq. (7) with or without the quantum noise included. Also in this case, Eq. (7) provides an accurate estimate across the entire spectrum. Notably, at this level of pump noise, quantum noise contributes significantly to the total $R_{S}$ in the DWG wavelength range,
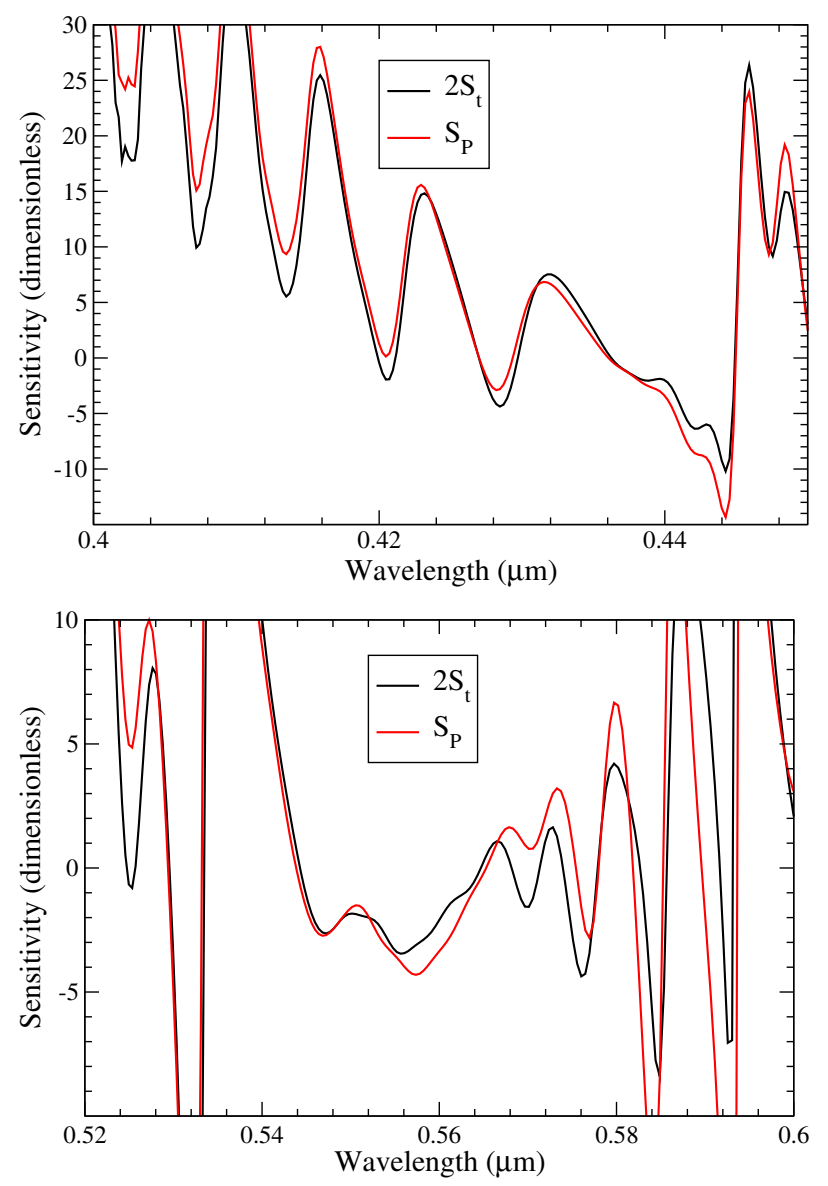

Fig. 8. Pump duration sensitivities $S_{t}$ (multiplied by 2) and pump power sensitivities $S_{P}$ in anomalous-dispersion fibers with $\Lambda=1.8 \mu \mathrm{m}$ (top) and $2.5 \mu \mathrm{m}$ (bottom) for a $100 \mathrm{fs}$ input pulse with $N=10$.

except at the short-wavelength side of the DWG peak. Here, pump noise is dominant and leads to quite strong relative fluctuations, more than an order of magnitude above the input pump noise level. The $N$ value of 10 is too low for the pulse to break up by modulational instability; nevertheless, quantum noise does affect the output somewhat due to the sensitive and "turbulent" nature of the pulse compression process. In general, the dispersive wave noise level is magnified several times over the pump noise level, except at the very peak of the DWG spectrum. Figure 10 shows a similar plot for pump relative duration and power fluctuations of $R_{t}=R_{P}=0.05$. There is a noticeable difference between the ensemble-averaged relative signal fluctuation $R_{S}$ and the one predicted by Eq. (7); thus, this is a case where the quadratic approximation is breaking down. The true $R_{S}$ function is seen to be smoother than that predicted by Eq. (7), especially the dips in noise level associated with zerocrossings of $R_{P}$ or $R_{t}$ are absent; however, the predicted noise peaks are also somewhat reduced. Overall, Eq. (7) still predicts the magnitude of the noise reasonably well.

Figure 11 shows the pump power and duration quantum equivalence fluctuations $R_{P q}, R_{t q}$ for $\Lambda=1.8 \mu \mathrm{m}$ and $\Lambda=2.5 \mu \mathrm{m}$ and $N=10$. While the fluctuations are somewhat larger than for the normal-dispersion case, it appears that a technical noise level around $10^{-3}$ is also desirable for DWG at 

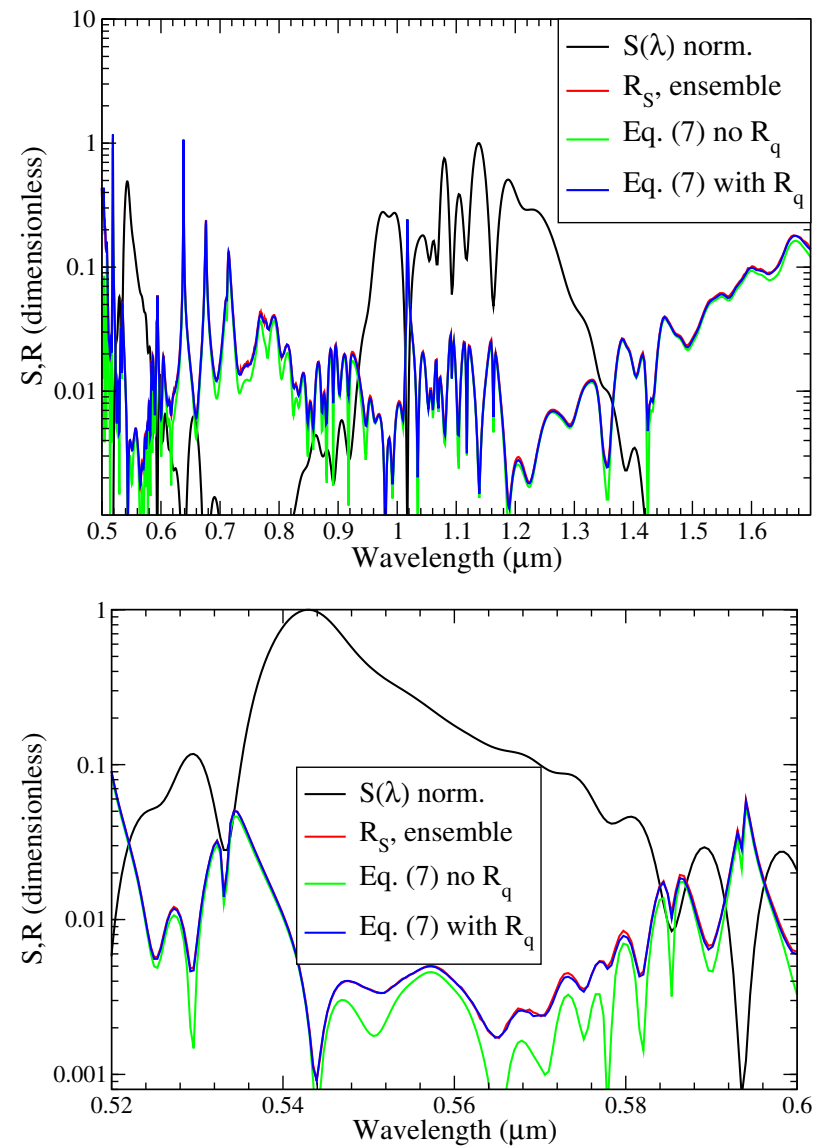

Fig. 9. Same as Fig. 4 for DWG with $\Lambda=2.5 \mu \mathrm{m}, N=10$, and independent relative pump power and duration fluctuations $R_{P}=R_{t}=10^{-3}$. The lower panel zooms in on the DWG wavelength range.

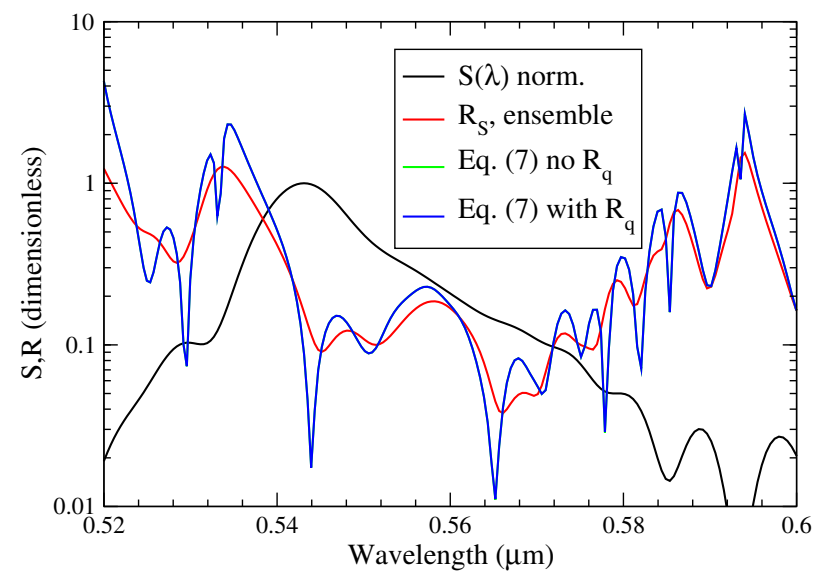

Fig. 10. Same as Fig. 9 lower panel, except that relative pump power and duration fluctuations $R_{P}=R_{t}=0.05$.

this power level (note that the spectrum for $\Lambda=2.5 \mu \mathrm{m}$ peaks just above $0.54 \mu \mathrm{m}$, and that for $\Lambda=1.8 \mu \mathrm{m}$ is large between 0.42 and $0.44 \mu \mathrm{m})$. Apparently, the somewhat larger pump power and duration sensitivities $S_{P}$ and $S_{t}$, compared with the normal-dispersion case, are offset by a correspondingly larger magnitude of quantum-noise-induced fluctuations.
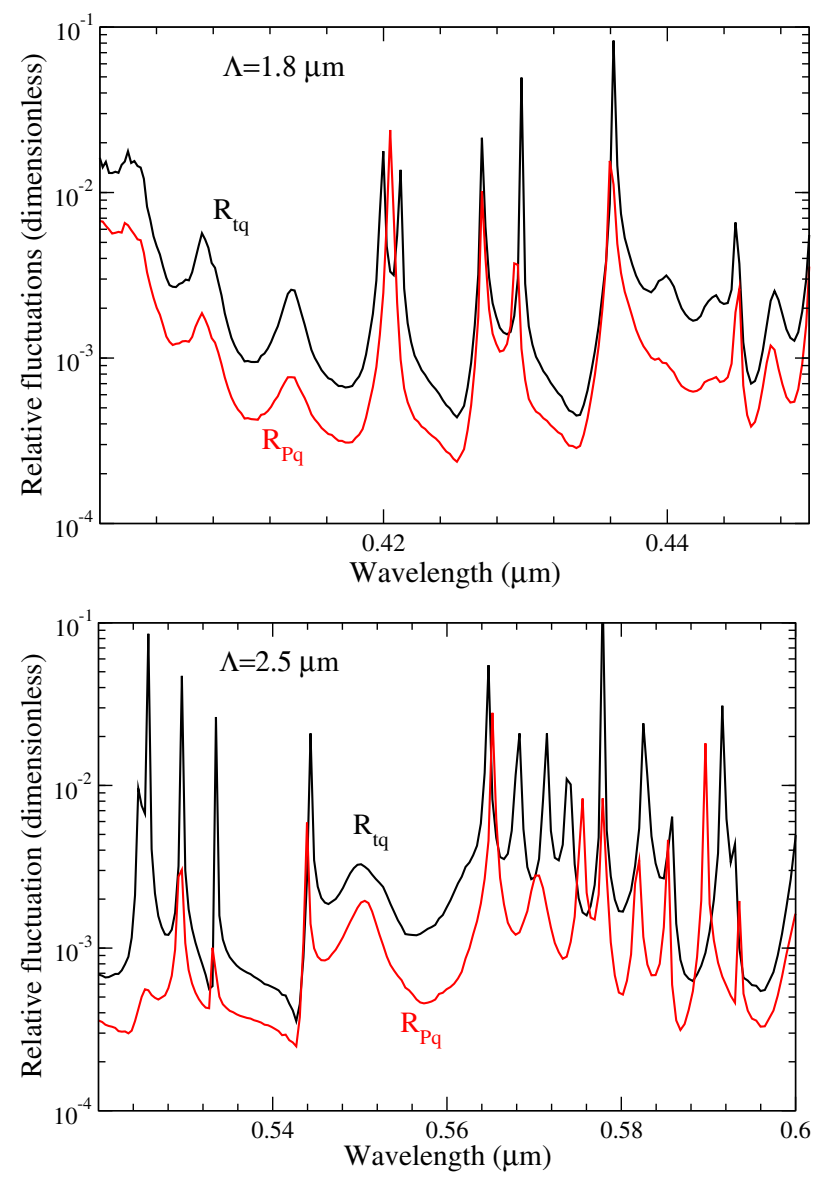

Fig. 11. Pump power and duration quantum equivalence fluctuations $R_{P q}$ and $R_{t q}$ as defined by Eq. (8) for DWG with $N=10$, $\Lambda=1.8 \mu \mathrm{m}$ (top) and $2.5 \mu \mathrm{m}$ (bottom).

\section{CONCLUSION}

In conclusion, a dimensionless measure of technical noise impact on nonlinear frequency conversion processes in optical fibers has been presented and tested in the two important cases of normal-dispersion supercontinuum generation and dispersive wave generation. The measure appears to predict the noise properties well for pump noise in the percent or per mill range. While one should be careful when drawing general conclusions from a small set of simulations, it is interesting that, in both cases studied, fundamental quantum noise effects begin to dominate if the technical noise drops substantially below the per mill level.

\section{APPENDIX A: DERIVATION OF BASIC EQUATION FOR $R_{S}$}

This appendix provides a brief derivation of Eq. (4). Assuming weak parameter variations, for a particular realization $m$ of the input parameters $p$, the value of the observable $S, S_{m}$ is expanded through second order in the deviations of $p$ from their mean values, $\delta p_{m}$ :

$$
S_{m} \approx S_{0}+\sum_{p}\left\{\frac{\partial S}{\partial p} \delta p_{m}+\frac{1}{2} \sum_{p^{\prime}} \frac{\partial^{2} S}{\partial p \partial p^{\prime}} \delta p_{m} \delta p_{m}^{\prime}\right\}+\delta S_{q m} .
$$


The term $\delta S_{q m}$ describes an additional zero-mean fluctuation from quantum noise effects, which is assumed independent of the fluctuations from noise in pump pulse parameters. When averaging over the ensemble of realizations, the assumptions of independent variations between different parameters and zero mean of $\delta p_{m}, \delta S_{q m}$ lead to

$$
\begin{gathered}
\left\langle S_{m}\right\rangle=\frac{1}{M} \sum_{m} S_{m}=S_{0}+\frac{1}{2} \sum_{p} \frac{\partial^{2} S}{\partial p^{2}} \delta p^{2} \\
\left\langle S_{m}^{2}\right\rangle=\frac{1}{M} \sum_{m} S_{m}^{2}=S_{0}^{2}+\delta S_{q}^{2} \\
+\frac{1}{M} \sum_{m} \sum_{p p^{\prime}}\left\{\frac{\partial S}{\partial p} \frac{\partial S}{\partial p^{\prime}} \delta p_{m} \delta p_{m}^{\prime}+S_{0} \frac{\partial^{2} S}{\partial p \partial p^{\prime}} \delta p_{m} \delta p_{m}^{\prime}\right\} \\
=S_{0}^{2}+\left(\delta S_{q}\right)^{2}+\sum_{p}\left\{\left(\frac{\partial S}{\partial p}\right)^{2}+S_{0} \frac{\partial^{2} S}{\partial p^{2}}\right\} \delta p^{2},
\end{gathered}
$$

where terms higher than second order in the $\delta p_{m}$ are omitted for consistency as well as products of $\delta S_{q m}^{2}$ and $\delta p_{m}^{2}$, which imply an assumption of weak quantum fluctuations as well. The parameters $\delta p \equiv \sqrt{\left\langle\delta p_{m}^{2}\right\rangle}$ and $\delta S_{q} \equiv \sqrt{\left\langle\delta S_{q m}^{2}\right\rangle}$ have also been introduced. The equivalence of the latter to the definition in Eq. (5) follows immediately from Eq. (A3) when setting all $\delta p$ to zero. Squaring Eq. (17) while casting away terms higher than second order in the $\delta p$ and subtracting the result from Eq. (A3) yields

$$
\left\langle S_{m}^{2}\right\rangle-\left\langle S_{m}\right\rangle^{2}=\delta S_{q}^{2}+\sum_{p}\left(\frac{\partial S}{\partial p}\right)^{2} \delta p^{2},
$$

i.e., the mean square deviation of $S$ in fact only depends on the first-order derivatives $\frac{\partial S}{\partial p}$. Equation (4) follows straightforwardly by taking the square root and dividing by $S_{0}$.

It may be noted that the assumption of zero mean for the $\delta S_{q m}$ distribution, strictly speaking, implies that $S_{0}$ should be calculated as the average of the quantum ensemble simulation rather than from a simulation without quantum noise. This was not done in the present work and so does not seem important for weak quantum noise, which is anyway assumed in the derivations. For situations with strong quantum noise, invalidating first- and second-order expansions, technical noise in the pump is likely to be less relevant to the total output noise.

Disclosures. The author declares no conflicts of interest.

Data Availability. Data underlying the results presented in this paper are not publicly available at this time but may be obtained from the authors upon reasonable request.

\section{REFERENCES}

1. J. K. Ranka, R. S. Windeler, and A. J. Stentz, "Visible continuum generation in air-silica microstructure optical fibers with anomalous dispersion at $800 \mathrm{~nm}$," Opt. Lett. 25, 25-27 (2000).

2. I. Cristiani, R. Tediosi, L. Tartara, and V. Degiorgio, "Dispersive wave generation by solitons in microstructured optical fibers," Opt. Express 12, 124-135 (2004).

3. J. E. Sharping, M. Fiorentino, P. Kumar, and R. S. Windeler, "Optical parametric oscillator based on four-wave mixing in microstructure fiber," Opt. Lett. 27, 1675-1677 (2002).

4. A. M. Heidt, A. Hartung, G. W. Bosman, P. Krok, E. G. Rohwer, H. Schwoerer, and H. Bartelt, "Coherent octave spanning near-infrared and visible supercontinuum generation in all-normal dispersion photonic crystal fibers," Opt. Express 19, 3775-3787 (2011).

5. B. Povazay, K. Bizheva, A. Unterhuber, B. Hermann, H. Sattmann, A. F. Fercher, W. Drexler, A. Apolonski, W. J. Wadsworth, J. C. Knight, P. St.J. Russell, M. Vetterlein, and E. Scherzer, "Submicrometer axial resolution optical coherence tomography," Opt. Lett. 27, 1800-1802 (2002).

6. J. M. Dudley, G. Genty, and S. Coen, "Supercontinuum generation in photonic crystal fiber," Rev. Mod. Phys. 78, 1135-1184 (2006).

7. K. L. Corwin, N. R. Newbury, J. M. Dudley, S. Coen, S. A. Diddams, K. Weber, and R. S. Windeler, "Fundamental noise limitations to supercontinuum generation in microstructure fiber," Phys. Rev. Lett. 90, 113904 (2003).

8. S. T. Sørensen, C. Larsen, U. Møller, P. M. Moselund, C. L. Thomsen, and $\mathrm{O}$. Bang, "Influence of pump power and modulation instability gain spectrum on seeded supercontinuum and rogue wave generation," J. Opt. Soc. Am. B 29, 2875-2885 (2012).

9. S. T. Sørensen, C. Larsen, U. Møller, P. M. Moselund, C. L. Thomsen, and $\mathrm{O}$. Bang, "The role of phase coherence in seeded supercontinuum generation," Opt. Express 20, 22886-22894 (2012).

10. J. Lægsgaard, "Scaling relations for soliton compression and dispersive-wave generation in tapered optical fibers," J. Opt. Soc. Am. B 35, 783-789 (2018).

11. X. Liu, A. S. Svane, J. Lægsgaard, H. Tu, S. A. Boppart, and D. Turchinovich, "Progress in Cherenkov femtosecond fiber lasers," J. Phys. D 49, 023001 (2016).

12. E. Genier, P. Bowen, T. Sylvestre, J. M. Dudley, P. Moselund, and O. Bang, "Amplitude noise and coherence degradation of femtosecond supercontinuum generation in all-normal-dispersion fibers," J. Opt. Soc. Am. B 36, A161-A167 (2019).

13. A. I. Adamu, M. S. Habib, C. R. Smith, J. E. A. Lopez, P. Jepsen, R. Amezcua-Correa, O. Bang, and C. Markos, "Noise and spectral stability of deep-UV gas-filled fiber-based supercontinuum sources driven by ultrafast mid-IR pulses," Sci. Rep. 10, 4912 (2020).

14. J. M. Dudley and S. Coen, "Coherence properties of supercontinuum spectra generated in photonic crystal and tapered optical fibers," Opt. Lett. 27, 1180-1182 (2002).

15. J. Lægsgaard, "Mode profile dispersion in the generalised nonlinear Schrödinger equation," Opt. Express 15, 16110-16123 (2007).

16. Z. Zhu and T. G. Brown, "Full-vectorial finite-difference analysis of microstructrured optical fibres," Opt. Express 10, 853-864 (2002).

17. A. M. Heidt, "Pulse preserving flat-top supercontinuum generation in all-normal dispersion photonic crystal fibers," J. Opt. Soc. Am. B 27, 550-559 (2010).

18. N. Akhmediev and M. Karlsson, "Cherenkov radiation emitted by solitons in optical fibers," Phys. Rev. A 51, 2602-2607 (1995).

19. K. F. Mak, J. C. Travers, P. Hölzer, N. Y. Joly, and P. St.J. Russell, "Tunable vacuum-UV to visible ultrafast pulse source based on gas-filled Kagome-PCF," Opt. Express 21, 10942-10953 (2013).

20. G. P. Agrawal, Nonlinear Fiber Optics (Academic, 2007). 